\title{
Isolated Finding of Intermittent Compression of the Trochlear Nerve by a Dolichoectatic Basilar Artery
}

\author{
(D) Ezgi Akar1, (D) Tamer Altay² \\ ${ }^{1}$ Haydarpaşa Numune Training and Research Hospital, Clinic of Neurosurgery, İstanbul, Turkey \\ 2University of Health Sciences Turkey, Prof. Dr. Cemil Taşçığlu City Hospital, Clinic of Neurosurgery, Istanbul, Turkey
}

\section{Abstract}

Vertebrobasilar dolichoectasia (VBD) is a rare disorder characterized by elongation and tortuosity of the vertebrobasilar arteries. Clinical manifestations can range from ischemic stroke and progressive compression of cranial nerves and brainstem to cerebral hemorrhage and hydrocephalus. Here, we report a case of intermittent diplopia complaint when looking down with a tilted head position for 3 months. His neuro-ophthalmic examination was within normal limits. Further, magnetic resonance and angiographic images revealed a dolichoectatic basilar artery compressing the right lateral brainstem. VBD treatment is controversial; there is still no effective treatment and only complications can be treated. Surgical options may be useful in very limited situations, but advances in stent technology are promising in the treatment of VBD. To our knowledge, no case in the literature describes intermittent trochlear nerve compression by VBD.

Keywords: Basilar artery, dolichoectasy, intermittent, ophthalmoplegia

\section{INTRODUCTION}

The term dolichoectasia is derived from "dolichos" and "ectasia", meaning elongation and dilatation (1). Vertebrobasilar dolichoectasia (VBD) is a rare disorder that is characterized by the elongation and tortuosity of the vertebrobasilar arteries affecting the neural elements and the brainstem (1), and it is commonly associated with the basilar artery (2). Based on the data obtained from the results of angiography and autopsy, the overall incidence of VBD is $<0.05 \%$ but can differ among ethnic groups (1). VBD parameters are as follows: Basilar or vertebral artery diameter $>4.5 \mathrm{~mm}$, deviation of any portion of either artery $>10 \mathrm{~mm}$ from the shortest expected course, or basilar and vertebral artery length $>29.5$ and $>23.5 \mathrm{~mm}$, respectively (2). Clinical manifestations of VBD include ischemic stroke, progressive compression of cranial nerves and brainstem, cerebral hemorrhage, and hydrocephalus. Midbrain symptoms are generally caused by ischemia or local compression (3). Ocular motor nerve paresis has been reported with recurrent trochlear nerve paresis being remarkably rare (3). Herein, we present an usual case of isolated trochlear nerve paresis associated with intermittent diplopia for just 30 seconds when looking down due to a dolichoectatic basilar artery.

\section{CASE PRESENTATION}

A 54-year-old man was admitted to our clinic complaining of intermittent diplopia for 3 months. He was tilting his head down for a few seconds when the vision problem started. He was on antihypertensive medication for 7 years, and his blood pressure had been within normal limits. He denied any other comorbidity. There was no history of trauma, headache, vertigo, or focal deficit.

During ophthalmological examination, visual acuity, color vision, pupil size, and reactivity were normal, and no ptosis, nystagmus, or eyelid twitch was observed. Fundus examination was bilaterally insignificant. Further, extraocular muscle functions

Phone: +902163868263 E-mail: ezgiaycicek@gmail.com ORCID ID: orcid.org/0000-0002-8582-2948

Cite this article as: Akar E, Altay T. Isolated Finding of Intermittent Compression of the Trochlear Nerve by a Dolichoectatic Basilar Artery. Eur Arch Med Res 2021;37(1):49-51

๑Copyright 2021 by the University of Health Sciences Turkey, Prof. Dr. Cemil Taşçığlu City Hospital

European Archives of Medical Research published by Galenos Publishing House. 
were normal, but he complained about vertical diplopia while reading three to four times a day for a few seconds. Magnetic resonance imaging (MRI) and magnetic resonance angiography (MRA) revealed a hypoplastic right vertebral artery and an elongated and tortuous left vertebral artery as well as widened basilar artery that was compressing the brainstem (Figure 1A-C).

\section{DISCUSSION}

Based on angiography and autopsy results, the overall incidence of $\mathrm{VBD}$ is $<0.05 \%$, but, there is currently no data available on the exact incidence of this disorder. According to a Japanese study of people undergoing routine MRI and MRA examination, the incidence of asymptomatic VBD is $1.3 \%(2,3)$. VBD is typically asymptomatic, but approximately $6 \%$ of patients have clinical findings.

In our case of trochlear nerve paresis associated with VBD, the patient was symptomatic with head position. Furthermore, the incidence of VBD is higher in stroke patients than in normal populations, particularly in those who had posterior system infarctions (3). According to the MRI and MRA criteria described by Giang et al. (4) and Ubogu and Zaidat (5), VBD is characterized by the following: A basilar or vertebral artery diameter $>4.5$ $\mathrm{mm}$, deviation of any portion of either arteries $>10 \mathrm{~mm}$ from the shortest expected course, basilar and vertebral artery length $>29.5$ and $>23.5 \mathrm{~mm}$, respectively (2), or intracranial vertebral artery diameter $>4 \mathrm{~mm}(4,5)$.

Clinical manifestations of VBD include ischemic stroke, progressive compression of cranial nerves and brainstem, cerebral hemorrhage, dizziness, ataxia, and hydrocephalus.
The present case was evaluated due to intermittent trochlear nerve paresis. Symptoms can often arise after 60 years, and the development of VBD and its symptoms are correlated to atherosclerotic factors (3). Midbrain symptoms are generally due to ischemia or local compression (2). Moreover, ocular motor nerve paresis has been reported, with recurrent trochlear nerve paresis being remarkably rare (3). Herein, we present an unusual case isolated trochlear nerve paresis associated with intermittent diplopia for just 30 seconds when looking down due to a dolichoectatic basilar artery. Although there are several cases of recurrent cranial nerve paresis associated with VBD, position-related intermittent trochlear paresis has not been reported.

Intracranial arterial malformations are frequently observed. The clinical significance of these malformations is that they can cause aneurysm and VBD and are often associated with stroke. The most common arterial malformation is fenestration and is mostly found in the vertebrobasilar system. The incidence of congenital VBD is unclear, but it is estimated to be low (6).

Although the etiology of VBD is not clear, a range of potential mechanisms have been proposed. VBD can be congenital, acquired, or both. Hypertension-induced atherosclerosis may be a major factor in the development of VBD (1). Other suspected conditions include infections; congenital diseases, such as autosomal recessive polycystic kidney disease, Pompe disease, Fabry disease, sickle cell anemia, Marfan syndrome, PHACES syndrome; abnormal matrix metalloproteinase expression; male sex; smoking; and drinking (2,7-10). Our patient had no history of trauma or any disease. Pathological assessment showed rarefaction of the elastic tissue in the media with degenerated
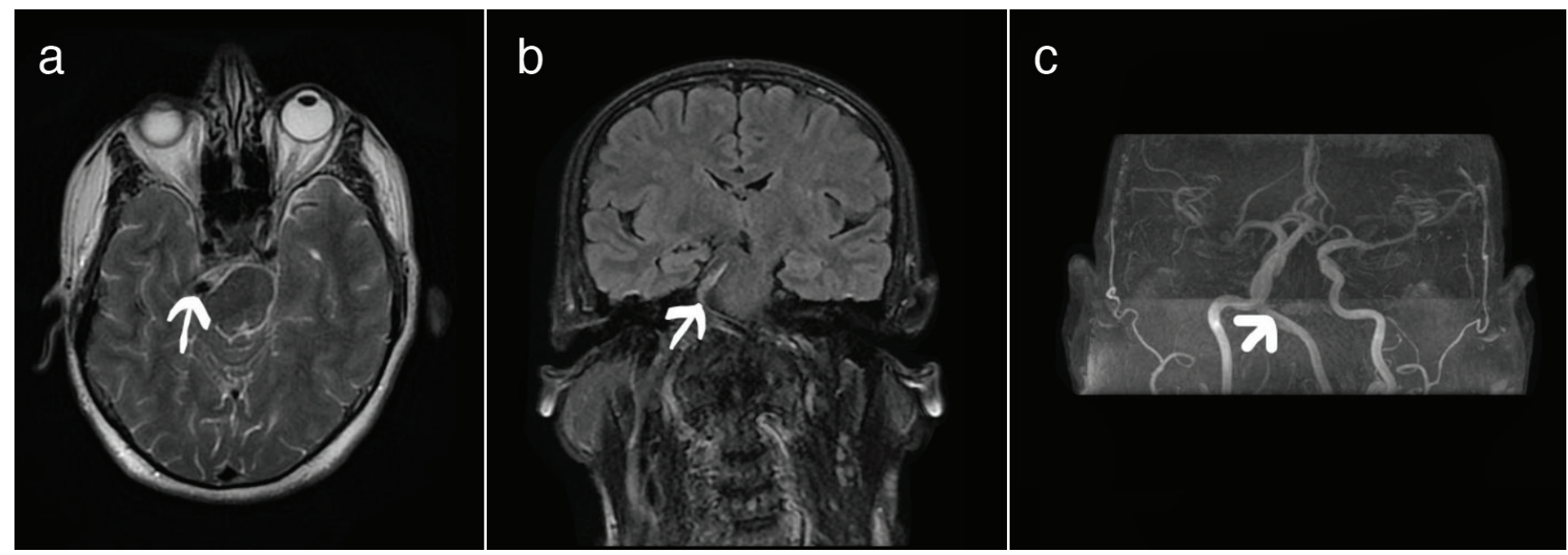

Figure 1. T1 axial magnetic resonance imaging shows a marked enlargement of the basilar artery and compression of pons (a). Widened basilar artery (b). MR angiography delineates the left vertebral artery as well as elongated and tortuous basilar artery (c) MR: Magnetic resonance 
internal elastic lamina and atrophy and reticular fiber deficiency in smooth muscle layer. Dolichoectatic arteries have an abnormally large external diameter with a thin arterial wall, sometimes containing thrombus or an atherosclerotic plaque in lumen $(1,3)$.

VBD treatment is still controversial and is not recommended for asymptomatic patients. In symptomatic VBD cases, treatment can be decided according to the findings. In patients with hydrocephalus, shunt surgery is performed, whereas microvascular decompression and stent surgery can be performed in patients with cranial nerve findings.

Anticoagulant therapy is recommended for stroke patients or patients who are at risk of stroke (3). We did not administer any medication to our patient who showed intermittent symptoms.

Isolated trochlear nerve paresis may be associated with many factors, such as diabetes, trauma, infectious diseases, meningitis, tumors, demyelinating disease, aneurysms, stroke, and complications of cranial surgery $(2,10)$, with trauma being the most common cause of isolated trochlear nerve paresis (2). Although there are many known causes of trochlear nerve paresis, VBD is very uncommon. Oculomotor nerve paresis is another known but rare finding. Persistent trochlear nerve paresis is even less common among patients, whereas, to our knowledge, intermittent paresis has not yet been reported (2).

Trochlear nerve paresis is associated with superior oblique muscle weakness, the clinical diagnosis of which is based on worsening vertical diplopia when reading or sitting at a table (2). Such patients must adopt a compensatory head posture. Accordingly, our patient compensated his double vision by tilting his head up.

\section{CONCLUSION}

In conclusion, isolated trochlear nerve paresis arising from VBD is a rare disorder. The findings related to the intermittent compression of the brainstem due to the basilar artery are exceptionally rare. Further, brain MRI and MRA are effective tools to identify elongation and dilatation of the vertebrobasilar system.

\section{Ethics}

Informed Consent: Was obtained as orally.

Peer-review: Externally peer-reviewed.

\section{Authorship Contributions}

Surgical and Medical Practices: T.A., Concept: E.A., T.A., Design: E.A., T.A., Data Collection or Processing: T.A., Analysis or Interpretation: E.A., T.A., Literature Search: E.A., Writing: E.A., T.A.

Conflict of Interest: No conflict of interest was declared by the authors.

Financial Disclosure: The authors declared that this study received no financial support.

\section{REFERENCES}

1. Dorland WA. The American illustrated medical dictionary. 1936;83:749.

2. Yuan YJ, Xu K, Luo Q, Yu JL. Research progress on vertebrobasilar dolichoectasia. Int J Med Sci 2014;11:1039-48.

3. Wang F, Hu XY, Wang T, Fang XM, Dai Z, Guo DL, et al. Clinical and imaging features of vertebrobasilar dolichoectasia combined with posterior circulation infarction: A retrospective case series study. Medicine (Baltimore) 2018;97:e13166

4. Giang DW, Perlin SJ, Monajati A, Kido DJ, Hollander J. Vertebrobasilar dolichoectasia: assessment using MR. Neuroradiology 1988;30:518-23.

5. Ubogu EE, Zaidat 00. Vertebrobasilar dolichoectasia diagnosed by magnetic resonance angiography and risk of stroke and death: a cohort study. J Neurol Neurosurg Psychiatry 2004;75:22-6.

6. Kovač JD, Stanković A, Stanković D, Kovač B, Šaranović D. Intracranial arterial variations: a comprehensive evaluation using CT angiography. Med Sci Monit 2014;20:420-7.

7. Kim HS, Choi $\mathrm{CH}$, Lee TH, Kim SP. Fusiform aneurysm presenting with cervical radiculopathy in ehlers-danlos syndrome. J Korean Neurosurg Soc 2010:48:528-31.

8. Mahadevan A, Tagore R, Siddappa NB, Santosh V, Yasha TC, Ranga U, et al. Giant serpentine aneurysm of vertebrobasilar artery mimicking dolichoectasia--an unusual complication of pediatric AIDS. Report of a case with review of the literature. Clin Neuropathol 2008;27:37-52.

9. Toyoshima Y, Emura I, Umeda Y, Fujita N, Kakita A, Takahashi H. Vertebral basilar system dolichoectasia with marked infiltration of IgG4-containing plasma cells: a manifestation of IgG4-related disease? Neuropathology 2012;32:100-4

10. Ortak H, Tas U, Aksoy DB, Ayan E. Isolated upgaze palsy in a patient with vertebrobasilar artery dolichoectasia; a case report. J Ophthalmic Vis Res 2014;9:109-12. 